\title{
Kleine Keime, große Wirkung
}

\section{Der Einfluss der Mikrobiota auf das Immunsystem}

Viele verbinden mit dem Begriff des Immunsystems primär den Thymus, das Knochenmark, die Rachenmandeln oder Lymphe. Seltener wird an den Darm gedacht. Dabei läuft ohne diesen immunologisch nicht allzu viel. Das ahnten schon unsere medizinischen Altvorderen, die im Darm weit mehr sahen als ein reines Verdauungsrohr. Sie kannten allerdings einen entscheidenden Grund dafür noch nicht: die zentrale Rolle seiner mikrobiellen Besiedlung, der Darmmikrobiota. Denn sie übernimmt für uns (über)lebenswichtige Aufgaben, darunter auch das Training unseres Immunsystems [1].

\section{Im Darm: die meisten Immunzellen}

Die immunologische Bedeutung des Darmes hat entscheidend mit seiner großen Fläche zu tun. Um eine effektive Verdauung und Resorption von Nährstoffen sicherzustellen, ist die Darmschleimhaut mit verschiedenen Oberflächenvergrößerungen ausgestattet. Zotten und Mikrovilli lassen über nur einige Meter die Schleimhautfläche auf vermutlich mehrere Hundert Quadratmeter anwachsen. Obwohl die Vergrößerung der Oberfläche wichtig ist für die Nährstoffversorgung, nimmt mit steigender Fläche die Gefahr zu, dass potenzielle Schadstoffe in den Körper gelangen. Die größte „grüne Grenze“ unseres Körpers bedarf daher eines besonderen Schutzes, der nicht nur durch

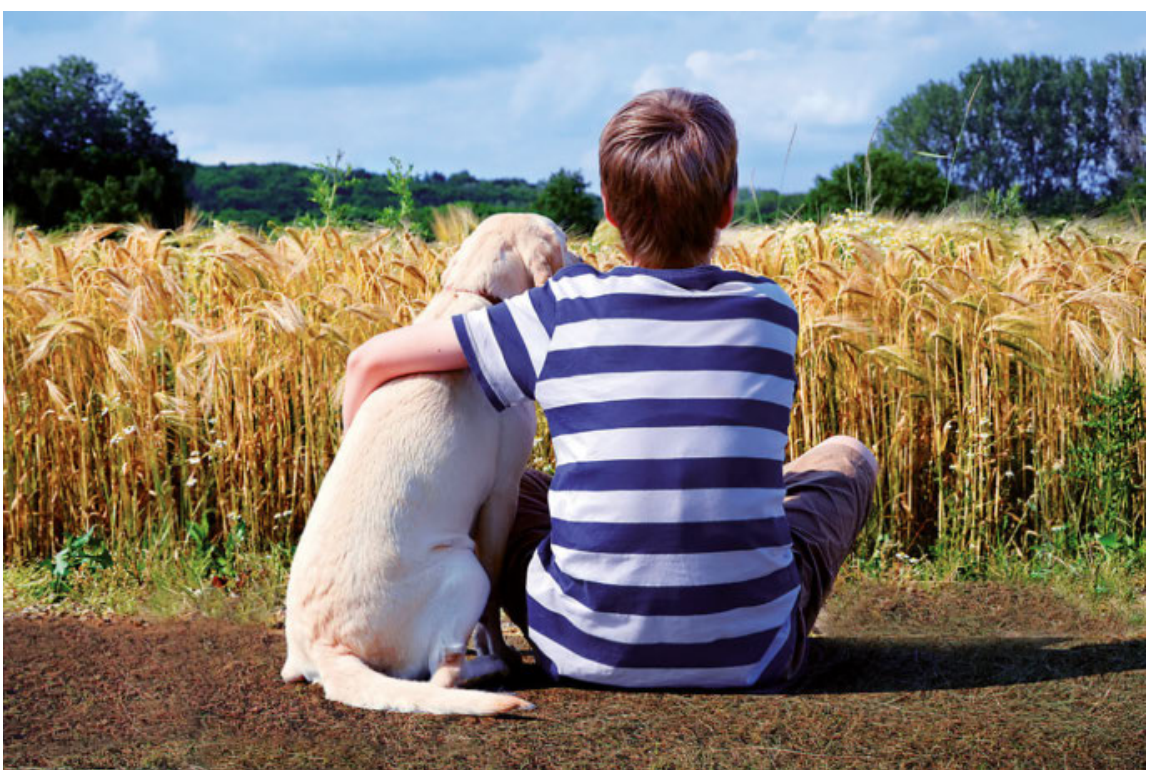

Abb. 1 Kindheit auf dem Land: Zusätzliche mikrobiologische Reize sorgen für immunologischen Schwung im Darm. (c) mekcar/Fotolia

die Integrität der Darmschleimhaut gewährleistet wird. Daran beteiligen sich auch die Darmmikrobiota sowie das darmassoziierte Immunsystem (GALT = Gut associated lymphoid tissue). Aufgrund der großen Darmoberfläche ist der Großteil, schätzungsweise $70-80 \%$ unserer Immunzellen, im Darm stationiert. Diese hohe immunologische Präsenz trägt nicht nur der vital notwendigen Abwehr von „Grenzverletzungen“ Rechnung. Der Darm ist auch der wichtigste Ausbildungsstandort für Immunzellen [2].

\section{Immunzentrale Darm}

Wie auch unsere Muskeln benötigt das Immunsystem ein permanentes Training, um nicht „einzurosten“. Die wichtigste immunologische „Muckibude“ liegt dabei im Darm. Ohne Vertragsbindung stehen uns dabei mit Vertretern unserer Darmmikrobiota rund um die Uhr qualifizierte „Personal Trainer“ zur Seite. Diese setzen nicht nur immunogene Signalsubstanzen wie kurzkettige Peptide frei. Auch Zellwandbestandteile der Mikroben selbst „kitzeln“ das Immunsystem. Allerdings bedarf es dazu spezialisierter Dolmet- 
Der Darm spielt als Hauptstandort der Immunzellen eine zentrale Rolle für die Funktionsfähigkeit der menschlichen Körperabwehr. Entscheidend ist dabei v.a. der Zustand der Darmmikrobiota. Bei chronischen Erkrankungen mit immunologischer Beteiligung, wie Infektanfälligkeit und Allergien ist daher auch der Status der Darmökologie zu berücksichtigen. Dieser kann über die Stuhldiagnostik ermittelt und dann gezielt u. a. mit pro- und präbiotischen Maßnahmen beeinflusst werden.

\section{Schlüsselwörter}

Darmmikrobiota, GALT, MALT, orale Toleranz, Stuhldiagnostik, Probiotika, Präbiotika, Synbiotika.

scher für die Kommunikation zwischen Mikroben und menschlichen Immunzellen. Als solche fungieren bspw. sog. Toll-like-Rezeptoren sowie bestimmte Darmepithelzellen, wie die M-Zellen (Microfold-Zellen). Letztere sind besonders im Bereich der Peyer-Plaques, im Dünndarm zu finden. Ihre Aufgabe: die Aufnahme und Konfektionierung immunogener Bestandteile aus dem Darminhalt sowie deren Weiterleitung an die Einrichtungen des darmassoziierten

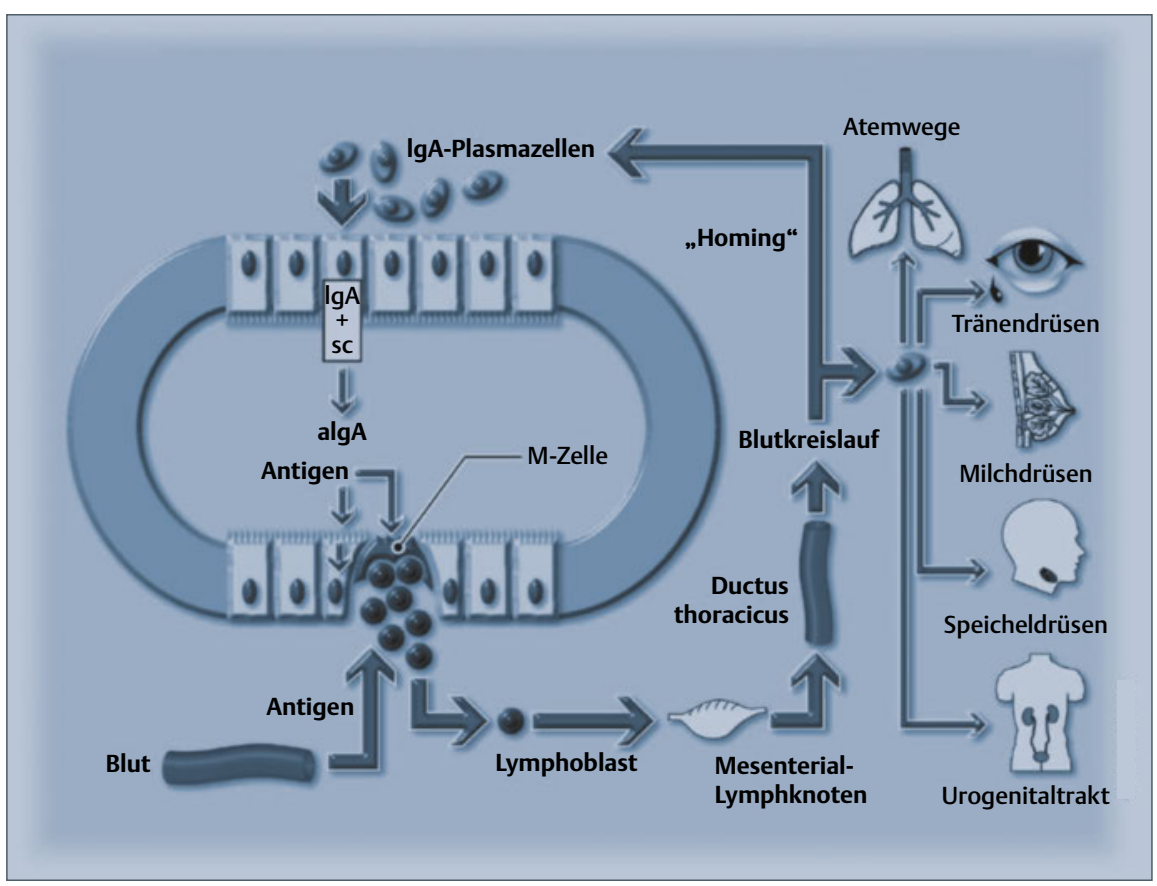

Abb. 2 Immunologische Vernetzung der Schleimhäute (aus [2] mod. nach [4]). (c) Labor L+S AG plays a central role for the functionality of the human immune system. An essential factor for this is the gut microbiota. Therefore in case of chronic diseases with immunological participation like susceptibility to infection and allergy the status of the gut ecology must be included. This can be done via stool diagnostic which allows to influence the gut specifically with probiotics and prebiotics.

\section{Keywords}

Gut microbiota, GALT, MALT, oral tolerance, stool diagnostic, probiotics, prebiotics, synbiotics.

Immunsystems in der Submukosa. Die dortigen Immunzellen werden mit diesen Reizen geschult [3].

Nach ausreichender immunologischer Lehre gehen viele Immunzellen auf Wanderschaft. Ihr Ziel sind die anderen Körperschleimhäute. Denn der Darm ist mit den anderen Körperoberflächen im sog. MALT (Mucosa associated lymphoid tissue) vernetzt (Abb. 2). Das Immuntraining im Darm beeinflusst deshalb auch die Abwehrlage z.B. in den Atemwegen
The gut as main harbour of immune cells

sowie im Urogenitaltrakt. Nur wenn es im Darm „rund läuft“, ist auch die Schlagkraft in der Peripherie gesichert. Ein Teil der vagabundierenden Immunzellen kehrt auch wieder in den Darm zurück. Dies wird als „Homing“ bezeichnet. Denn gerade auch im Darm bedarf es natürlich einer gut geschulten Abwehr [4].

\section{Wichtiger Abwehrfaktor: slgA}

Ein wichtiger Effektor der spezifischen Schleimhautabwehr wird von entsprechend trainierten Plasmazellen produziert und durch die Epithelzellen an die Oberfläche transportiert: das sekretorische Immunglobulin A (kurz: sIgA). Dieser spezielle Antikörper ist auf allen Schleimhäuten zu finden und fungiert dort als wichtiger erster „Abfangjäger“ für potenzielle Schadstoffe, wie Infektionserreger oder Allergene. Durch eine sog. sekretorische Komponente ist er im Gegensatz zu anderen Antikörpern vor dem mikrobiellen Abbau geschützt. Sonst würde er von der ortsansässigen Mikroflora schnell inaktiviert. Und noch eine Besonderheit zeichnet das sIgA aus: Die Antigen-Antikörper-Bindung führt $\mathrm{zu}$ keiner Komplementaktivierung. Damit bleibt das Anlocken von Abwehrzellen aus. Trotzdem arbeitet das sIgA sehr effektiv. Die gebundenen Schadstoffe werden am Kontakt mit der Darmschleimhaut gehindert und mit dem Stuhl entsorgt [5].

\section{Immunität und Immuntraining}

Auch das stammesgeschichtlich viel ältere, angeborene Immunsystem wird entscheidend von der Darmmikrobiota geprägt. Zu den Abwehrkomponenten, die bereits bei Reptilien nachweisbar sind, zählen die sog. Defensine, eine Gruppe breit antimikrobiell wirksamer Peptide. Diese werden von spezialisierten Darmzellen produziert und sorgen als „Abstandshalter" dafür, dass weder fremde noch Mikroorganismen der eigenen Flora der Darmschleimhaut zu sehr ,auf die Pelle“ rücken und in diese eindringen können. Bekanntester Vertreter im Darm ist das ß-Defensin 2. Dessen Bildung und Freisetzung wird durch die Darmmikrobiota induziert [6]. 
Besonders wichtig ist der Prozess des mikrobiellen Immuntrainings bei Kindern. Als Neugeborene kommen wir abwehrtechnisch gänzlich unbedarft auf die Welt. Für die nötige Sicherheit sorgt zunächst der hohe Gehalt an Abwehrfaktoren in der Muttermilch. Damit erhält das Kind einen allerdings zeitlich begrenzten „Nestschutz“. Spätestens mit dem Abstillen muss jedoch die eigene Abwehr lauffähig sein. Das erreicht sie nur, wie bereits erwähnt, durch die Auseinandersetzung mit der eigenen Darmmikrobiota. Diese wiederum nimmt ihren Ausgang von der mütterlichen Darm- und Vaginalflora. Erste maternale Keime treten dabei möglicherweise schon im Mutterleib über. Den größten Schwung an Mikroben erhält das Neugeborene allerdings bei der Geburt. Die dann abgeschluckten Keime legen die Basis für die kindliche Darmmikrobiota. Deren Entwicklung läuft parallel zur Entwicklung des Immunsystems sowie zu der Entwicklung der Darmschleimhaut. Dabei ist offensichtlich entscheidend, in welcher Reihenfolge die Keime ihren Platz im Säuglingsdarm finden $[7,8,9,10]$.

\section{Gefahr der immunologischen Fehlprogrammierung}

Störungen in der Darmbesiedlung können Auswirkungen haben, die weit über das Auftreten von bspw. Dreimonatskoliken hinausgehen. Denn in den ersten Lebensmonaten lernt das Immunsystem nicht nur die „Verteidigung“. Es lernt auch, gewisse Dinge zu tolerieren. Schließlich muss es zwischen problematischen Fremdstoffen wie Infektionserregern und lebensnotwendigen Nahrungsmitteln unterscheiden. Letztere dürfen nicht zu einer unproduktiven Abwehrreaktion führen. Für diese auch als „orale Toleranz“ bezeichnete Programmierung - sie ist noch nicht gänzlich verstanden - ist ein wesentlicher Faktor bekannt: die frühkindliche Darmmikrobiota. Fehlprogrammierungen aufgrund von mikrobiellen Störungen können zum erhöhten Allergierisiko führen [11].

\section{Beeinflussung der Darmbesiedlung}

Es lauern eine ganze Reihe an Faktoren, welche die normale Entwicklung der Darmbesiedlung torpedieren können. Seien es Störungen der mütterlichen Vaginalflora (Vaginosen, Vaginalmykosen), sei es die Geburt per Kaiserschnitt, die fehlende oder zu kurze Versorgung mit Muttermilch oder im frühen Lebensalter verabreichte Antibiosen. Tatsächlich können solche Störungen das Risiko der Kinder für Erkrankungen des allergischen Formenkreises erhöhen [12, 13, 14].

Dreck, Schnuller und Co.

Auch mikrobielle Reize aus der Umgebung können gerade bei Kindern für zusätzlichen immunologischen Schwung im Darm sorgen. Das zeigt z.B. der Vergleich zwischen Land- und Stadtkindern. Erstere haben ein deutlich niedrigeres Allergierisiko. Der Grund dafür ist offensichtlich nicht die schadstoffärmere, sondern die mikrobenreichere Umgebung [15]. Ein gewisses Maß an „Dreck“ gehört daher zu einer gesunden Kindheit dazu. Umso bedenklicher ist die Verbreitung stark antimikrobiell wirksamer Haushaltsreiniger. Insbesondere das lernende kindliche Immunsystem braucht Beschäftigung, um nicht auf „dumme Gedanken“ zu kommen. Eine übertriebene Hygiene mit einer mangelnden immunologischen Auslastung wird daher ernsthaft als ein Faktor für die Zunahme allergischer Erkrankungen sowie von Autoimmunerkrankungen, wie z.B. Typ-1-Diabetes diskutiert [16, 17, 18].

Dass ein orales Immuntraining bei Kindern offenbar sehr hilfreich ist, zeigen zwei auf den ersten Blick etwas kuriose Studien.

- In der ersten Untersuchung aus Schweden wurde ein Zusammenhang zwischen dem elterlichen Umgang mit dem Schnuller ihres Sprösslings sowie dessen Allergierisiko nachgewiesen. Dieses war am niedrigsten, wenn die Eltern den heruntergefallenen Schnuller einfach vor dem erneuten Gebrauch ableckten. Ein höheres Risiko bestand in den Familien, die den Schnuller vor dem weiteren Ge- brauch abgewaschen oder sogar abgekocht hatten [19].

- Eine zweite Studie zeigte bei Kindern in Neuseeland gegenüber einer Kontrollgruppe eine deutlich niedrigere allergische Sensibilisierung, wenn diese Daumen lutschten oder Nägel kauten [20].

Vielleicht müssten vor diesem Hintergrund doch einige pädagogische Regeln neu bewertet werden.

\section{Infektanfälligkeit}

Funktionsstörungen des GALT und eine beeinträchtigte Abwehrlage stehen sicherlich nicht vorrangig mit dem Gebrauch von Schnullern und Fingern im Zusammenhang. Neben Schädigungen der Darmschleimhaut oder dem Mangel an Nährstoffen ist in vielen Fällen das Ungleichgewicht der Darmmikrobiota ursächlich [21]. Die Gründe dafür reichen von falscher Ernährung bis hin zu iatrogenen Einflüssen wie Antibiosen. Letzteres meint insbesondere den häufig noch zu beobachtenden ungezielten Einsatz von Antibiotika, der die wichtige Kommunikation zwischen Mikrobiota und Immunsystem stören kann. Insbesondere bei Kindern kann dadurch ein folgenreicher Circulus vitiosus gebahnt werden: Das aufgrund eines Infektverdachts gegebene Antibiotikum trifft im besten Fall zwar den Infektionserreger. Allerdings erwischt es in der Regel auch Vertreter der körpereigenen Mikrobiota. Das mikrobielle Immuntraining gerät so ins Stottern - der nächste Infekt ist damit in vielen Fällen vorprogrammiert. Mit der erneuten Antibiose dreht sich die Spirale weiter. Das Risiko solcher Entwicklungen lässt sich am besten frühzeitig mit begleitenden probiotischen Maßnahmen vermindern.

\section{Allergien}

Doch die Folgen solcher „Beziehungsstörungen“ zwischen Darmmikrobiota und Mensch bzw. dem Immunsystem beschränken sich klinisch nicht auf die Infektanfälligkeit. Schon lange sind „Darmdysbiosen“ als wichtiger mitverursachender Faktor bei Erkrankungen des allergischen Formenkreises bekannt. Ob 
Tab. 1 Stuhlparameter zur Abklärung des Barrierezustands im Darm.

\begin{tabular}{|c|c|}
\hline Parameter & Aussage über \\
\hline Stuhlflora & Zustand der mikrobiellen Barriere im Darm \\
\hline$\alpha$-1-Antitrypsin, Zonulin & $\begin{array}{l}\text { Zustand der Darmschleimhaut-Barriere (erhöhte Durch- } \\
\text { lässigkeit, Leaky Gut?) }\end{array}$ \\
\hline $\begin{array}{l}\text { sekretorisches Immunglobu- } \\
\text { lin A }\end{array}$ & Zustand der immunologischen Darmbarriere \\
\hline ß-Defensin 2 & Zustand des angeborenen Darmimmunsystems \\
\hline
\end{tabular}

Asthma, Heuschnupfen oder Neurodermitis - die meisten Patienten weisen Störungen ihrer Darmökologie auf, auch wenn sich die klinische Symptomatik an ganz anderen Stellen äußert. Darüber hinaus steht die Darmmikrobiota - wie schon erwähnt - auch bei vielen Autoimmunerkrankungen als wichtiger immunologischer Einflussfaktor immer mehr im Fokus.

\section{Diagnostische Möglichkeiten}

Viele Gründe also, sich auch in der täglichen Praxis intensiv mit der Darmmikrobiota und ihrer therapeutischen Beeinflussung zu beschäftigen. Und das nicht nur bei offensichtlich im Darm begründeten Beschwerden, wie chronischen Bauchproblemen („Reizdarm“). Aufgrund seines zentralen Stellenwertes ist die Darmökologie bei fast allen chronischen Erkrankungen mit immunologischer Beteiligung sich am einfachsten über eine Untersuchung des Stuhles erfassen. Dabei haben sich verschiedene Parameter bewährt, die eine Aussage über die Darmmikrobiota, die Darmschleimhaut und das GALT gestatten (Tab. 1).

Bei der routinediagnostischen Untersusich die Analysen aktuell auf eine Reihe deren Bedeutung und therapeutische Beeinflussung bekannt und belegt ist. Aufwändige molekularbiologische Mikrobiom-Analysen bleiben derzeit wissenschaftlichen Fragestellungen vorbehalten. Sie bieten aufgrund des fehlenden Wissens zu den meisten dort bestimmten Keikennbaren klinischen Mehrwert.

\section{Therapie}

Der Darm ist nicht nur diagnostisch, sondern auch therapeutisch das ideale immunologische Zielorgan. Probiotika beeinflussen nicht nur die Darmmikrobiota positiv, sie fungieren auch als Immunmodulatoren $[22,23]$.

\section{Probiotika}

Als passende „Werkzeuge“ zur Beeinfluszu berücksichtigen. Deren Zustand lässt chung der Darmmikrobiota beschränken besonders prominenter Markerkeime, men zum jetzigen Zeitpunkt keinen er- sung von Darmmikrobiota und -immunsystem haben sich v.a. Probiotika erwiesen. Das sind laut WHO Zubereitungen, „die (lebende) Mikroorganismen enthalten, die in ausreichender Menge verabreicht, einen positiven Einfluss auf die Gesundheit des Wirtes haben“. Dazu werden vornehmlich Escherichia coli, Lactobacillus sp., Bifidobacterium sp., Enterococcus sp., Streptococcus thermophilus und Hefen der Spezies Saccharomyces cerevisiae verwendet. Bis auf die Streptokokken und den Saccharomyces sind dies Vertreter der normalen Darmikrobiota.

\section{Mikrobiologische Immuntherapie}

Die orale Gabe von Mikroorganismen führt zu einer verstärkten „Reizung“ der bereits beschriebenen Immunrezeptoren im Darm. Daraus resultiert allerdings nicht eine reine Abwehrsteigerung im Sin- 


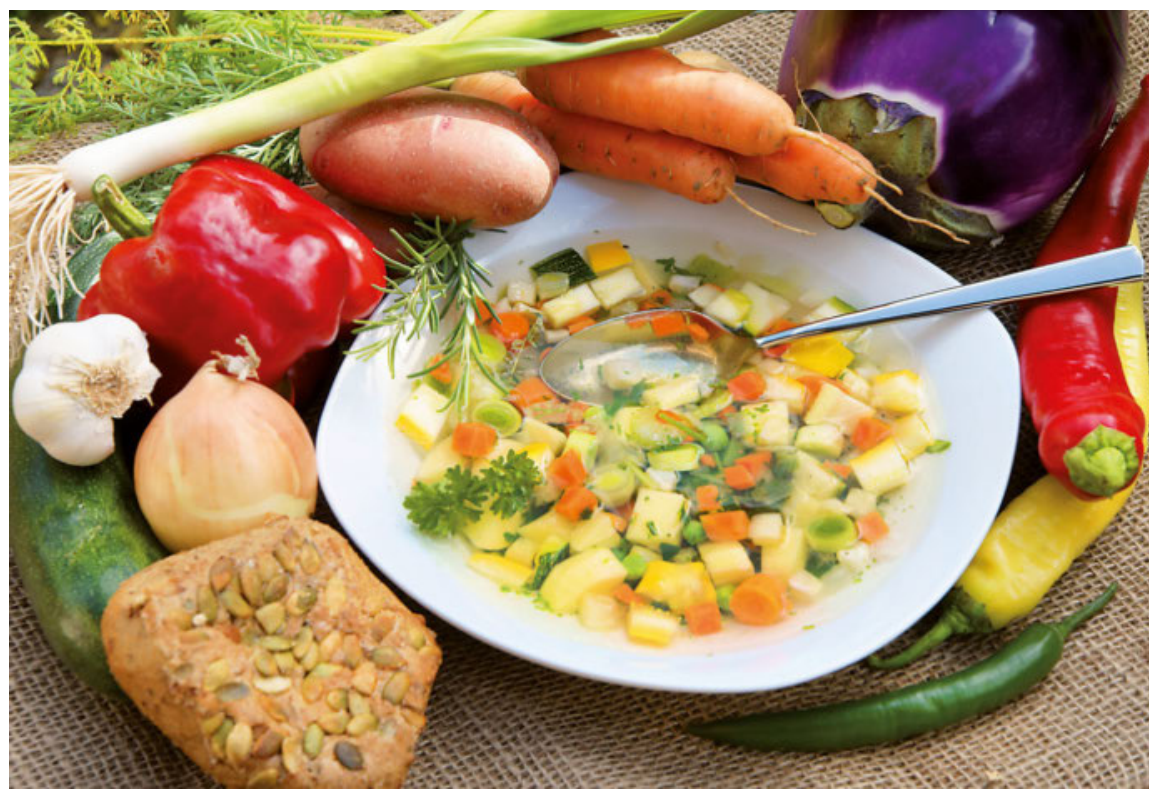

Abb. 3 Über die Ernährung kann nachhaltig auf die Darmmikrobiota Einfluss genommen werden - mit einem ausreichenden Gehalt an Ballaststoffen. () Johanna Mühlbauer/Fotolia

ne einer Immunstimulation. Probiotika sind vielmehr auch in der Lage, überschießende und unproduktive Reaktionen, wie sie im Rahmen von allergischen Geschehen ablaufen, zu dämpfen. Deshalb trifft der Begriff der „Immunmodulation“ die tatsächliche Wirkung besser.

Aufgrund der geschilderten Vernetzung der Schleimhautimmunsysteme ermöglicht die orale Probiose letztlich über den Darm auch eine elegante Verbesserung der Abwehrsituation bspw. in den Atemoder Harnwegen. Solche immunologischen Effekte sind übrigens auch mit nicht lebensfähigen Keimen oder deren Stoffwechselprodukten zu erreichen. Daraus folgt, dass die offizielle Probiotika-Definition eigentlich nicht weitreichend genug ist.

\section{Unterschiedliche Reizstärke beachten}

Die grundsätzliche immunologische Wirkung verschiedener probiotischer Keime ist offensichtlich vergleichbar und entspricht einer eher unspezifischen Immunmodulation. Allerdings unterscheiden sie sich in der Stärke des Immuneffektes. Diese sog. Immunogenität steigt von den mikrobiologischen Stoffwechselprodukten über Enterokokken, Laktobazillen und Bifidobakterien bis hin zu E. coli als stärkstem Immunmodulator an. Das macht sich teilweise auch bei der klinischen Verträglichkeit in Form anfänglicher Bauchbeschwerden bemerkbar.

\section{Praxis}

Insbesondere bei stärkeren Immunogenen wie $\mathrm{E}$. coli ist daher eine einschleichende Dosierung empfehlenswert. Entscheidend für die Wirksamkeit ist zudem eine regelmäßige Einnahme über einen ausreichend langen Zeitraum. Dieser sollte i. d. R. mindestens 2-3 Monate betragen.

\section{Arznei- und Nahrungsergänzungs- mittel}

Die konkrete Auswahl eines Probiotikums erfolgt am besten auf Basis eines Stuhlflorabefunds, der die gezielte Beeinflussung der Mikrobiota mit einem geeigneten Präparat ermöglicht. Es stehen sowohl Arzneimittel als auch sog. Nahrungsergänzungsmittel zur Verfügung. Ein wichtiger formaler Unterschied liegt in der Erstattungsfähigkeit.

\section{- Probiotische Arzneimittel werden}

u.U. von privaten Krankenversicherungen und bei Kindern bis 12 Jahren sowie unter bestimmten Voraussetzungen bei Colitis ulcerosa (Remissionserhaltung bei Unverträglichkeit von Aminosalicylaten) teilweise auch von den gesetzlichen Krankenversicherungen erstattet.

- Nahrungsergänzungsmittel müssen selbst bezahlt werden.

Außerdem sind bestimmte Keime, wie z.B. lebende Escherichia coli nur als Arzneimittel verfügbar. Das liegt daran, dass in Nahrungsergänzungsmitteln nur Keime eingesetzt werden dürfen, die traditionell zur Lebensmittelgewinnung verwendet werden. Das sind insbesondere Laktobazillen, Bifidobakterien und $\mathrm{He}$ fen. Für die klinische Wirkung ist dieser formale Unterschied allerdings unerheblich.

Wichtig für den therapeutischen Effekt ist dagegen die verwendete Keimzahl. Üblicherweise sollte eine therapeutische Dosis für Erwachsene mindestens $10^{8}-10^{9}$ Keime enthalten. Diese Voraussetzung erfüllen fast alle am Markt befindlichen Präparate.

\section{Keine guten oder schlechten Probiotika}

Insofern gibt es auch keine guten oder schlechten Probiotika. Es gibt nur weniger gut oder besser individuell zum Patienten passende Präparate. Ausschlaggebend ist der Stuhlflora-Befund. Zu berücksichtigen sind ferner pragmatische Gesichtspunkte wie z.B. die Formulierung oder der Preis. Um die Auswahl eines geeigneten Produktes zu erleichtern, ist über den Autor eine unabhängige Übersicht der verfügbaren probiotischen Arznei- und Nahrungsergänzungsmittel erhältlich. Auch die Anzahl und Art, der in einem Probiotikum enthaltenen verschiedenen Spezies, ist offensichtlich für die klinische Wirkung nicht wirklich ausschlaggebend. Entscheidend ist die Gesamtzahl z.B. an Laktobazillen oder Bifidobakterien.

\section{Ernährung und Präbiotika}

Doch nicht nur durch die Zufuhr von Mikroorganismen lassen sich die Darmmikrobiota und das Immunsystem des Darms positiv regulieren. Auch über die Ernährung kann nachhaltig auf die Darmmikrobiota Einfluss genommen werden. Ausschlaggebend dafür ist v.a. der ausreichende Gehalt an Ballaststoffen. Die- 
se fördern das Wachstum bzw. die Aktivität verschiedener Vertreter unserer Darmmikrobiota. Daraus resultiert indirekt auch eine Beeinflussung des darmassoziierten Immunsystems. Sehr konzentriert sind solche Wirkungen über den Einsatz von Präbiotika erreichbar Ballaststoffkonzentrate wie Inulin, resistente Maisstärke und Flohsamenschalen aber auch der künstliche Zweifachzucker Lactulose. Werden diese präbiotischen Substanzen mit Mikroorganismen kombiniert, werden entsprechende Präparate als Synbiotika bezeichnet.

\section{Fazit}

Wenn es um die Gesundheit geht, führt kein Weg am Darm und seiner Mikrobiota vorbei. Das gilt insbesondere für eine „schlagkräftige“ Immunabwehr. Doch erst in den letzten Jahren findet dieses der Naturheilkunde integrale Wissen zunehmend die ihm gebührende Berücksichtigung in der konventionellen Medizin. Die Stuhldiagnostik sowie darauf basierende pro-, prä- und synbiotischen Strategien bieten dabei für die tägliche Praxis effektive Maßnahmen zur positiven Beeinflussung der Darmmikrobiota sowie des Immunsystems.

Online zu finden unter:

http://dx.doi.org//10.1055/s-0042-123661

Interessenkonflikte: Der Autor ist Mitarbeiter der Labor L+S AG/Enterosan, die auch Stuhldiagnostik anbietet.

\section{Literatur}

[1] Wilson M. Microbial Inhabitants of Humans. Their Ecology and Role in Health and Disease. Cambridge: Cambridge University Press; 2005

[2] Beckmann G, Rüffer A. Mikroökologie des Darmes. Grundlagen, Diagnostik, Therapie. Bad Bocklet: Eigenverlag Labor L+S AG; 2016

[3] Ivanov II, Honda K. Intestinal commensal microbes as immune modulators. Cell Host Microbe 2012; 12 (4): 496-508
[4] Gebbers JO, Laissue JA. Das intestinale Immunsystem. Teil I: Funktionelle Aspekte. Med Klin 1984; 79: 13-19

[5] Brandtzaeg P. Homeostatic impact of indigenous microbiota and secretory immunity. Ben Microbes 2010; 1: 211-227

[6] Wehkamp J, Bals R, Kreft B et al. Angeborene Immunität. Dtsch Ärztebl 2007; 104: A257-262

[7] Fanaro S, Chierici R, Guerrini $\mathbf{P}$ et al. Intestinal microflora in early infancy: composition and development. Acta Paediatr Suppl. 2003; 91: 48-55

[8] Isolauri E. Development of healthy gut microbiota early in life. J Paed Child Health 2012; 48 (Suppl 3): 1-6

[9] Schindlbeck K. Die Bedeutung des Geburtsmodus für die Entwicklung der intestinalen Mikrobiota Neugeborener. [MasterArbeit]. Fachhochschule Salzburg: 2015

[10] Dominguez. B.llomg, Costello EK, Contreras $M$ et al. Delivery mode shapes the acquisition and structure of the initial microbiota across multiple body habitats in newborns. Proc Natl Acad Sci USA 2010; 107: 1197111975

[11] Hougteling PD, Walker AW. From birth to 'immunohealth', allergies and enterocolitis. I Clin Gastroenterol 2015; 49: 7-12

[12] Benn CS, Thorsen P, Jensen JS et al. Vaginal microflora during pregnancy and the risk of asthma hospitalization and use of antiasthma medication in early childhood. J Allergy Clin Immunol 2002; 110: 72-77

[13] Bennet R, Nord CE. Development of the faecal anaerobic microflora after caesarean section and treatment with antibiotics in newborn infants. Infection 1987; 15: 332336

[14] Neu J, Rushing J. Cesarean versus vaginal delivery: long-term infant outcomes and the hygiene hypothesis. Clin Perinatol 2011; 38: 321-331

[15] Lynch SV et al. Effects of early-life exposure to allergens and bacteria on recurrent wheeze and atopy in urban children. J Allergy Clin Immunol 2014; 134: 593-601

[16] Rook GAW. Hygiene hypothesis and autoimmune diseases. Clin Rev Allergy Immunol 2012; 42: 5-15

[17] Mori K, Nakagawa Y, Ozaka H. Does the gut microbiota trigger Hashimoto's thyroiditis? Discov Med 2012; 14: 321-326

[18] Parekh PJ, Arusi E, Vinik Al et al. The role and influence of gut microbiota in patho- genesis and management of obesity and metabolic syndrome. Front Endocrinol 2014; 5: 472-169

[19] Thompson JC, Dolen WK. Pacifier cleaning practices and risk of allergy development. Pediatr 2014; 134 (Suppl 3): S136-S137

[20] Lynch S], Sears MR, Hancox RJ. Thumbsucking, nail-biting, and atopic sensitization, asthma, and hay fever. Pediatrics 2016; 138 (2): e20160443

[21] Ege M], Mayer M, Normand AC et al. Disruption of the gut microbiome as a risk factor for microbial infections. Curr Opin Microbiol 2013; 16: 221-227

[22] Bischoff SC, Hrsg. Probiotika, Präbiotika und Synbiotika. Stuttgart: Thieme; 2009

[23] Schulze J, Sonnenborn U, Ölschläger T et al. Probiotika: Mikrökologie, Mikrobiologie, Qualität, Sicherheit und gesundheitliche Effekte. Stuttgart: Hippokrates; 2008

\section{ÜBER DEN AUTOR}

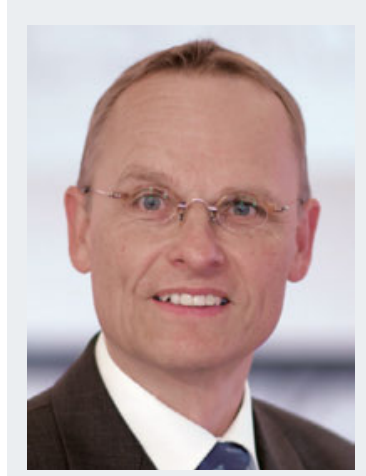

Andreas Rüffer ist Fachtierarzt für Mikrobiologie. Nach dem Studium der Veterinärmedizin war er als wissenschaftlicher Mitarbeiter am Institut für Mikrobiologie der Tierärztlichen Hochschule Hannover tätig. Seit 1994 leitet er den Bereich „Enterosan ${ }^{\circledR} /$ Klinische Mikroökologie“ der Labor L+S AG.

KORRESPONDENZADRESSE

Dr. Andreas Rüffer

Labor L+S AG/Enterosan ${ }^{\circledR}$

Mangelsfeld 4, 5, 6

97708 Bad Bocklet-Großenbrach

E-Mail: Andreas.Rueffer@Labor-LS.de 